\title{
Unsteady Magnetohydrodynamic Heat Transfer in a Semi-Infinite Porous Medium with Thermal Radiation Flux: Analytical and Numerical Study
}

\author{
O. Anwar Bég, ${ }^{1}$ J. Zueco, ${ }^{2}$ S. K. Ghosh, ${ }^{3}$ and Alireza Heidari ${ }^{4}$ \\ ${ }^{1}$ Aerospace Engineering, Department of Engineering and Mathematics, Sheaf Building, \\ Sheffield Hallam University, Sheffield S1 1WB, UK \\ ${ }^{2}$ Thermal Engineering and Fluids Department, Technical University of Cartagena, \\ Campus Muralla del Mar, 30202 Cartagena, Spain \\ ${ }^{3}$ Magnetohydrodynamics, Applied Mathematics Program, Department of Mathematics, \\ Narajole Raj College, P.O. Narajole, Midnapore 721 211, WB, India \\ ${ }^{4}$ Institute for Advanced Studies, Tehran 14456-63543, Iran
}

Correspondence should be addressed to O. Anwar Bég, docoanwarbeg@hotmail.co.uk

Received 12 December 2010; Accepted 20 January 2011

Academic Editor: William J. Layton

Copyright (C) 2011 O. Anwar Bég et al. This is an open access article distributed under the Creative Commons Attribution License, which permits unrestricted use, distribution, and reproduction in any medium, provided the original work is properly cited.

The unsteady, buoyancy-induced, hydromagnetic, thermal convection flow in a semi-infinite porous regime adjacent to an infinite hot vertical plate moving with constant velocity, is studied in the presence of significant thermal radiation. The momentum and energy conservation equations are normalized and then solved using both the Laplace transform technique and Network Numerical Simulation. Excellent agreement is obtained between both analytical and numerical methods. An increase in Hartmann number $\left(M^{2}\right)$ strongly decelerates the flow and for very high strength magnetic fields $\left(M^{2}=20\right)$, the flow is reversed after a short time interval. The classical velocity overshoot is also detected close to the plate surface for low to intermediate values of $M^{2}$ at both small and large times; however this overshoot vanishes for larger strengths of the transverse magnetic field $\left(M^{2}=10\right)$. An increase in radiation-conduction parameter $\left(K_{r}\right)$ significantly increases temperature throughout the porous regime at both small and larger times, adjacent to the plate, but decreases the shear stress magnitudes at the plate. Temperature gradient is reduced at the plate surface for all times, with a rise in radiation-conduction parameter $\left(K_{r}\right)$. Shear stress is reduced considerably with an increase in Darcian drag parameter $\left(K_{p}\right)$.

\section{Introduction}

Transient MHD (magnetohydrodynamic) flows with and without heat transfer in electricallyconducting fluids have attracted substantial interest in the context of metallurgical fluid dynamics, re-entry aerothermodynamics, astronautics, geophysics, nuclear engineering, 
and applied mathematics. An early study was presented by Carrier and Greenspan [1] who considered unsteady hydromagnetic flows past a semi-infinite flat plate moving impulsively in its own plane. Gupta [2] considered unsteady magneto-convection under buoyancy forces. Singer [3] further assessed the unsteady free convection heat transfer with magnetohydrodynamic effects in a channel regime. Pop [4] reported on transient buoyancy-driven convective hydromagnetics from a vertical surface. Yu and Yang [5] investigated the influence of channel wall conductance on hydromagnetic convection. Rao [6] analyzed the unsteady magnetohydrodynamic convection heat transfer past an infinite plane. Further excellent studies of unsteady free convection magnetohydrodynamic flows were reported by Antimirov and Kolyshkin [7] for a vertical pipe and Rajaram and Yu for a parallel-plate channel [8]. Tokis [9] used Laplace transforms to analyze the threedimensional free-convection hydromagnetic flow near an infinite vertical plate moving in a rotating fluid when the plate temperature undergoes a thermal transient. The influence of oscillatory pressure gradient on transient rotating hydromagnetic flow was considered by Ghosh [10]. Other transient MHD studies include the papers by Sacheti et al. [11], Attia [12] who included viscosity variation effects, Al-Nimr and Alkam [13] who considered open-ended vertical annuli, and Takhar et al. [14] who employed a numerical method to study flat-plate magnetohydrodynamic unsteady convection flow. Eswara et al. [15] examined the transient laminar magnetohydrodynamic convection in a cone due to a point sink, with the free stream velocity varying continuously with time and also for the case of an impulsive change either in the strength of the point sink or in the wall temperature. They showed numerically that magnetic field increases the skin friction but decreases heat transfer and that the transient nature of the convection flow is active for short durations with suction present and greater times with injection. Chamkha [16] has analyzed the unsteady MHD free three-dimensional convection over an inclined permeable surface with heat generation/absorption. Jha [17] presented exact solutions for transientfree convection MHD Couette channel flow with impulsive motion of one of the plates discussed. More recent communications on unsteady hydromagnetic heat transfer flows include the articles by Seddeek [18] incorporating variable viscosity effects, Zakaria [19] who considered a polar fluid, and Ghosh and Pop [20] who included Hall currents [21]. Zueco presented network simulation solutions for the transient natural convection MHD flow with viscous heating effects. Bég et al. [22] studied the free convective MHD flow from a spinning sphere with impulsive motion using the Blottner difference method. Duwairi et al. [23] analyzed the unsteady MHD natural convection for the non-Boussinesq case that is, using a nonlinear density relationship for water at low temperatures. Bozkaya and Tezer-sezgin [24] have presented boundary element numerical solutions for transient magnetohydrodynamic flow in a rectangular duct with insulating walls, showing that $n$ increase in Hartmann number causes the formation of boundary layers for both the velocity and the induced magnetic field with the velocity becoming uniform at duct centre. With increasing magnetic field, the time for reaching steady-state solution is also reduced. In many industrial applications, hydromagnetic flows also occur at very high temperatures in which thermal radiation effects become significant. The vast majority of radiation-convection flows have utilized algebraic flux approximations to simplify the general equations of radiative transfer [25]. The most popular of these simplifications remains the Rosseland diffusion approximation which has been employed by for example, Ali et al. [26] and later by Hossain et al. [27]. Radiation magnetohydrodynamic convection flows are also important in astrophysical and geophysical regimes. Raptis and Massalas [28] considered induced magnetic field effects in their study of unsteady hydromagnetic-radiative free convection. 


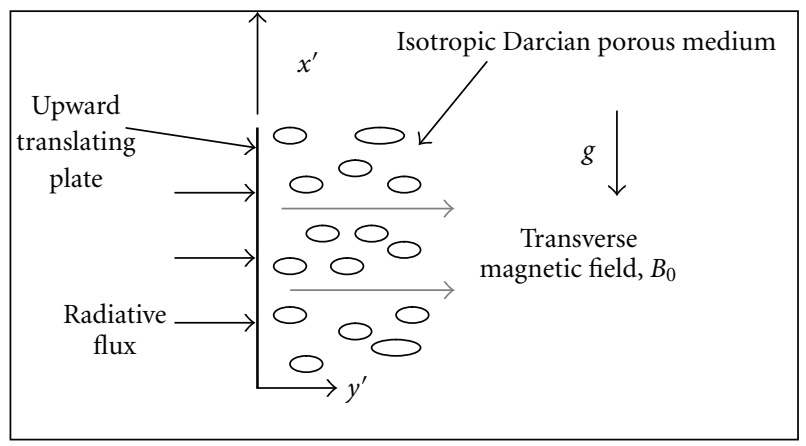

Figure 1: Physical regime and coordinate system.

Abd El-Naby et al. [29] numerically studied magnetohydrodynamic (MHD) transient natural convection-radiation boundary layer flow with variable surface temperature, showing that velocity, temperature, and skin friction are enhanced with a rise in radiation parameter increases, whereas Nusselt number is reduced. Ogulu and Motsa [30] studied the transient radiation-magnetohydrodynamic Couette flow in a channel with wall conductance variation, showing that heat transfer rates are boosted with a reduction in Hartmann number when the wall is nonconducting, but conversely increase with a rise in values of the magnetic Reynolds number at a given value of electrical conductance. Abd-El Aziz [31] studied the thermal radiation flux effects on unsteady MHD micropolar fluid convection. Ogulu and Prakash [32] obtained analytical solutions for variable suction and radiation effects on dissipative-free convective, optically-thin, magnetohydrodynamic flow using a differential approximation to describe the radiative flux. More recent studies involving thermal radiation and transient hydromagnetic convection include the analyses by Prasad et al. [33] which included species transfer and Zueco [34] who also considered viscous heating. In numerous geophysical and metallurgical flows, porous media may also arise. Classically the Darcian model is used to simulate the bulk effects of porous materials on flow dynamics and is valid for Reynolds numbers based on the pore radius, up to approximately 10. Chamkha [35] studied the transient-free convection magnetohydrodynamic boundary layer flow in a fluid-saturated porous medium channel, and later [36] extended this study to consider the influence of temperature-dependent properties and inertial effects on the convection regime. Bég et al. [37] presented perturbation solutions for the transient oscillatory hydromagnetic convection in a Darcian porous media with a heat source present. Chaudhary and Jain [38] studied the influence of oscillating temperature on magnetohydrodynamic convection heat transfer past a vertical plane in a Darcian porous medium. In the present study, we shall consider the transient radiation-convection magnetohydrodynamic flow past a vertical plate adjacent to a Darcian regime. Both analytical and numerical solutions have been obtained.

\section{Mathematical Model}

Consider the unsteady free convective hydromagnetic flow of an incompressible, viscous incompressible, electrically-conducting fluid along an infinite hot vertical plate moving with constant velocity, adjacent to a saturated porous regime. The physical scenario is shown in Figure 1. The $x^{\prime}$-axis is oriented along the plate from the leading edge in the vertically 
upward direction, and the $y^{\prime}$-axis is perpendicular to this. A uniform magnetic field, $B_{0}$, is applied parallel to the $y^{\prime}$-axis that is, transversely to the plate. Thermal radiation acts as a unidirectional flux in the $y^{\prime}$-direction. The fluid is gray and absorbing-emitting but nonscattering, and the magnetic Reynolds number is assumed to be small enough to neglect induced magnetic field effects. Hall and ionslip current effects are also neglected. The electromotive force generated by a magnetic field is a function of the speed of the fluid and the magnetic field strength. Following Shercliff [39], we define the electrical field intensity, E, using Maxwell's equation:

$$
\nabla \times E=-\frac{\partial B}{\partial t^{\prime}}
$$

where $t^{\prime}$ is time. The magnetic flux density, $B$, is defined as follows:

$$
B=\mu E \mathrm{H},
$$

where $H$ is the magnetic field strength and $\mu_{e}$ is the magnetic permeability. The generalized Ohm's law defines the total current flow as follows:

$$
J=\sigma(E+V \times B)
$$

where $V$ is the velocity vector and $\sigma$ is the electrical conductivity of the fluid. The electromagnetic retarding force, $F_{\text {magnetic, }}$ to be incorporated into the momentum conservation equation, then takes the form:

$$
F_{\text {magnetic }}=J \times B=\sigma(V \times B) \times B \text {. }
$$

Incorporating this magnetic retarding force in the momentum boundary layer equation, the appropriate conservation equations, under the Boussinesq approximation for the flow under the above assumptions, may be expressed as

$$
\begin{gathered}
\frac{\partial u^{\prime}}{\partial x^{\prime}}+\frac{\partial v^{\prime}}{\partial y^{\prime}}=0 \\
\frac{\partial u^{\prime}}{\partial t^{\prime}}=v \frac{\partial^{2} u^{\prime}}{\partial y^{\prime 2}}+g \beta\left(T^{\prime}-T_{\infty}^{\prime}\right)-\frac{v u^{\prime}}{K}-\frac{\sigma B_{0}^{2}}{\rho} u^{\prime} \\
\frac{\partial T^{\prime}}{\partial t^{\prime}}=\frac{k_{1}}{\rho C_{p}} \frac{\partial^{2} T^{\prime}}{\partial y^{\prime 2}}-\frac{1}{\rho C_{p}} \frac{\partial q_{r}}{\partial y^{\prime}}
\end{gathered}
$$

The appropriate boundary conditions at the wall and in the free stream are

$$
\begin{gathered}
u^{\prime}=0, \quad T^{\prime}=T_{\infty}^{\prime} \quad \text { for } y^{\prime} \geq 0, t^{\prime} \leq 0, \\
u^{\prime}=U, \quad T^{\prime}=T_{w}^{\prime} \quad \text { for } y^{\prime}=0, t^{\prime}>0, \\
u^{\prime}=0, \quad T^{\prime} \longrightarrow T_{\infty}^{\prime} \quad \text { for } y^{\prime} \longrightarrow \infty
\end{gathered}
$$


where $u^{\prime}$ is the velocity along the plate, $v^{\prime}$ is velocity normal to the plate, $v$ is the kinematic viscosity of the conducting fluid, $g$ denotes gravity, $\beta$ is the coefficient of thermal expansion, $T^{\prime}$ is fluid temperature, $T_{\infty}^{\prime}$ is free stream temperature, $k_{1}$ is thermal conductivity of the fluid, $C_{p}$ is the specific heat capacity, $\rho$ is the fluid density, $q_{r}$ is radiative heat flux, $K$ is the permeability of the porous regime, $B_{0}$ the component of magnetic field in the $x^{\prime}$-direction, $T_{w}^{\prime}$ is the plate temperature (isothermal), and $U$ is the velocity of the plate. Following Isachenko et al. [40], we employ a diffusion-type radiation heat transfer approximation, namely,

$$
q_{r}=-\frac{4 \sigma^{*}}{3 k^{*}} \frac{\partial T^{\prime}}{\partial y^{\prime}}
$$

where $\sigma^{*}$ and $k^{*}$ are respectively the Stefan-Boltzmann constant and the spectral mean absorption coefficient of the saturated medium. Assuming that the temperature differences within the saturated porous regime are sufficiently small such that $T^{\prime 4}$ may be expressed as a linear function of the temperature, a power-series expansion of $T^{\prime 4}$ about $T_{\infty}^{\prime}$, neglecting higher order terms leads to

$$
T^{\prime 4}=4 T_{\infty}^{\prime 3} T^{\prime}-3 T_{\infty}^{\prime 4}
$$

Implementing (2.8) and (2.9) in (2.6), we arrive at the modified energy conservation equation:

$$
\frac{\partial T^{\prime}}{\partial t^{\prime}}=\frac{k_{1}}{\rho C_{p}} \frac{\partial^{2} T^{\prime}}{\partial y^{\prime 2}}+\frac{1}{\rho C_{p}} \frac{4 \sigma^{*}}{3 k^{*}} \frac{\partial^{2} T^{\prime 4}}{\partial y^{\prime 2}}
$$

In order to render solutions to the boundary value problem described by (2.5) and (2.10) subject to the spatial and temporal conditions specified in (2.7), we introduce a group of nondimensional transformations, defined as follows:

$$
\begin{aligned}
& u=\frac{u^{\prime}}{U}, \quad y=\frac{y^{\prime} U}{v}, \quad t=\frac{t^{\prime} U^{2}}{v}, \quad \theta=\frac{T^{\prime}-T_{\infty}^{\prime}}{T_{w}^{\prime}-T_{\infty}^{\prime}}, \quad P_{r}=\frac{\rho v C_{p}}{k_{1}}, \\
& G_{r}=\frac{g \beta v\left(T_{w}^{\prime}-T_{\infty}^{\prime}\right)}{U^{3}}, \quad K_{r}=\frac{16 \sigma^{*} T_{\infty}^{\prime 3}}{3 k^{*} k_{1}}, \quad K_{p}^{2}=\frac{g v}{K U^{2}}, \quad M^{2}=\frac{\sigma B_{0}^{2} v}{\rho U^{2}},
\end{aligned}
$$

where $u$ is dimensionless $x^{\prime}$-direction velocity, $y$ is dimensionless coordinate normal to the plane, $t$ is dimensionless time, $\theta$ is dimensionless temperature, $P_{r}$ is Prandtl number, $G_{r}$ is Grashof number, $K_{r}$ is the radiation-conduction parameter, $K_{p}^{2}$ is the Darcian drag force coefficient (inverse permeability parameter), and $M^{2}$ is the Hartmann magnetohydrodynamic 
parameter. The transformed equations are thereby reduced to the following pair of coupled, second-order partial differential equations:

$$
\begin{gathered}
\frac{\partial u}{\partial t}=\frac{\partial^{2} u}{\partial y^{2}}+G_{r} \theta-K_{p}^{2} u-M^{2} u \\
\left(1+K_{r}\right) \frac{\partial^{2} \theta}{\partial y^{2}}-P_{r} \frac{\partial \theta}{\partial t}=0 .
\end{gathered}
$$

The corresponding transformed boundary conditions become

$$
\begin{aligned}
& u=0, \quad \theta=0 \quad \text { for } y \geq 0, t \leq 0, \\
& u=1, \quad \theta=1 \quad \text { for } y=0, t>0, \\
& u=0, \quad \theta \longrightarrow 0 \quad \text { for } y \longrightarrow \infty .
\end{aligned}
$$

We note that the optically-thick radiative approximation is valid for relatively low values of the parameter, $K_{r}$. The electrically nonconducting version (i.e., with $M^{2}=0$ ) of (2.12) has recently been studied by Ghosh and Bég [41] where extensive computations were provided of the influence of thermal radiation on the flow field. In the present study, we shall consider the supplementary influence of transverse magnetic field for the case where the fluid is saturated with air for which the Prandtl number is assumed to take the value 0.7.

\section{Analytical Solution}

The Laplace transform technique is now employed to generate closed-form solutions for the coupled, linear partial differential equations (2.12) and (2.13) subject to the boundary conditions (2.14). The solutions for the transient velocity $(u)$ and transient temperature $(\theta)$ take the following expressions:

$$
\begin{aligned}
u(y, t)= & \frac{1}{2}\left(1-\frac{G_{r}}{D^{2}}\right)\left[\exp (-D y) \operatorname{erfc}\left(\frac{y-2 D t}{2 \sqrt{t}}\right)+\exp (D y) \operatorname{erfc}\left(\frac{y+2 D t}{2 \sqrt{t}}\right)\right] \\
& +\frac{G_{r}}{D^{2}} \operatorname{erfc}\left(\frac{y}{2} \sqrt{\frac{P_{r}}{\left(1+K_{r}\right) t}}\right)+\frac{G_{r}}{D^{2}} \frac{y}{2 \sqrt{\pi}} \frac{(\sqrt{2}-1) t^{-1 / 2}}{2} \\
& \times\left[\exp \left\{-\left(\frac{y^{2}}{4 t}+D^{2} t\right)\right\}-\sqrt{\frac{P_{r}}{1+K_{r}}} \exp \left(-\frac{y^{2}}{4 t} \frac{P_{r}}{1+K_{r}}\right)\right], \\
\theta(y, t)= & \operatorname{erfc}\left(\frac{y}{2} \sqrt{\frac{P_{r}}{\left(1+K_{r}\right) t}}\right) .
\end{aligned}
$$


The spatial gradients of these functions provide expressions for the dimensionless shear stress (i.e., related to the skin friction) and temperature gradient (i.e., related to the Nusselt number) at the plate surface:

$$
\begin{aligned}
\left.\frac{\partial u}{\partial y}\right|_{y=0}= & \frac{1}{2}\left(1-\frac{G_{r}}{D^{2}}\right)\left[(-D) \operatorname{erfc}(-D \sqrt{t})+(D) \operatorname{erfc}(D \sqrt{t})-\frac{2}{\sqrt{t \pi}} \exp \left(-D^{2} t\right)\right] \\
& -\frac{G_{r}}{D^{2}} \frac{1}{\sqrt{\pi}}\left[\sqrt{\frac{P_{r}}{\left(1+K_{r}\right) t}}\right]+\frac{G_{r}}{D^{2}} \frac{1}{4 \sqrt{\pi}}(\sqrt{2}-1) t^{-\frac{1}{2}}\left[\exp \left\{-D^{2} t\right\}-\sqrt{\frac{P_{r}}{1+K_{r}}}\right], \\
\left.\frac{\partial \theta}{\partial y}\right|_{y=0}= & -\frac{1}{\sqrt{\pi}}\left(\sqrt{\frac{P_{r}}{\left(1+K_{r}\right) t}}\right)
\end{aligned}
$$

in which $D^{2}=M^{2}+K_{p}^{2}$.

\section{Network Numerical Solution}

Numerical solutions to the two-point boundary value problem have also been obtained with the Network Simulation Method (NSM). This powerful and robust computational method has been employed extensively by the authors in a wide spectrum of both linear and nonlinear steady and unsteady magnetohydrodynamic and thermal convection flows. Zueco [42] recently studied the periodic temperature variation effect on thermal convection in a horizontal channel. Other very recent studies employing NSM include the works of Bég et al. [43] considered unsteady rotating Couette flow in a porous medium channel, [44] analyzed the magnetohydrdoynamic rotating flow in a Darcian channel with dissipation effects and Hall/ionslip currents, and [45] examined the effects of thermal stratification and non-Darcian drag on natural convection boundary layers in a porous regime. In the NSM technique, a second-order central-difference scheme is utilized to discretize the momentum and energy conservation equations and the resulting system of finite difference equations are solved employing the Pspice program [46]. A network model is subsequently designed, with component equations which are formally equivalent to the discretized ones. The electrical analogy relates the electrical current $(J)$ with the velocity flux $(\partial u / \partial y)$ and temperature flux $(\partial \theta / \partial y)$, while the electrical potential $(\Phi)$ is equivalent to the velocity, $u$ and temperature $\theta$. A number of networks are connected in series to make up the whole medium. After experimenting with a few sets of mesh sizes, a region of integration of 200 cells has been selected. Boundary conditions are subsequently added by means of special electrical devices (current or voltage control-sources) that is, resistors, capacitors, and so forth. Once the complete network model is designed, the Pspice code is employed for the numerical simulations. This code is designated the "electric circuits simulator". Using the Fourier Law, the spatial discretization of (2.12) and (2.13) gives

$$
\begin{gathered}
\frac{\Delta y d u_{i}}{d t}=\frac{\left(u_{i-\Delta y}-u_{i}\right)}{(\Delta y / 2)}-\frac{\left(u_{i}-u_{i+\Delta y}\right)}{(\Delta y / 2)}+G_{r} \theta_{i} \Delta y-\Delta y K_{p}^{2} u_{i}-\Delta y M^{2} u_{i}, \\
\frac{\Delta y P_{r}}{\left(1+K_{r}\right)} \frac{d \theta_{i}}{d t}=\frac{\left(\theta_{i-\Delta y}-\theta_{i}\right)}{(\Delta y / 2)}-\frac{\left(\theta_{i}-\theta_{i+\Delta y}\right)}{(\Delta y / 2)} .
\end{gathered}
$$




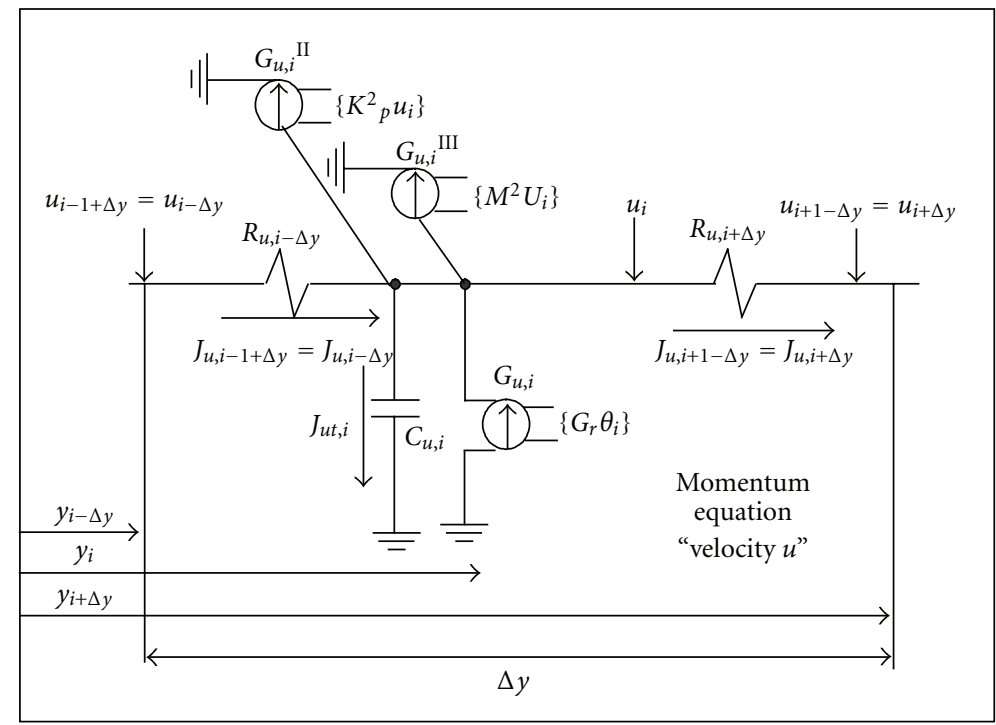

Figure 2: Electronic network model for the momentum equation (2.12).

The electrical analogy is applied to (4.1) together with Kirchhoff's law for the currents. To implement the boundary conditions at $y=0$ and $y=1$, constant voltage sources are employed for both conditions. The principal advantage of the NSM approach is that it avoids the necessity in traditional numerical difference schemes of manipulation of difference equations and the specified constraints concerning the convergence of numerical solutions. For example, the time-step used in transient problems, which is required for convergence is not a prerequisite as Pspice achieves this via sophisticated numerical algorithms largely analagous to those intrinsic to the standard difference numerical solvers, as described by Nagel [47]. Design of the model does require a comprehensive appreciation of electrical circuit theory. Momentum balance "currents" are defined systematically for each of the discretized equations and errors can be quantified in terms of the quantity of control volumes. The network model is shown in Figure 2 for the momentum equation (2.12) and Figure 3 for the energy equation (2.13).

\section{Results and Discussion}

We have obtained a comprehensive range of solutions to the transformed conservation equations. To test the validity of our numerical NSM computations, we have compared the velocity and shear stress distributions in Tables 1 and 2 with the Laplace transform solutions. Very good correlation is apparent. In all computations the key thermophysical parameters have been prescribed as follows, unless otherwise stated: $G_{r}=10, K_{r}=2.0$, $K_{p}=1.0, M^{2}=5.0, P_{r}=0.7$, and $t=0.25$, corresponding to free convection of air in a highly porous regime with strong magnetic field and high thermal radiation flux at intermediate time. Both Tables 1 and 2 correspond to distributions computed a short time after the initiation of motion that is, at $t=0.2$. In Table 1 , we observe that an increase in Hartmann number $\left(M^{2}\right)$ from 5.0 through 8 to 10 (strong magnetic flux density) causes a significant decrease in the flow velocity, $u$ with distance normal to the plate surface into the boundary layer $(y)$. 


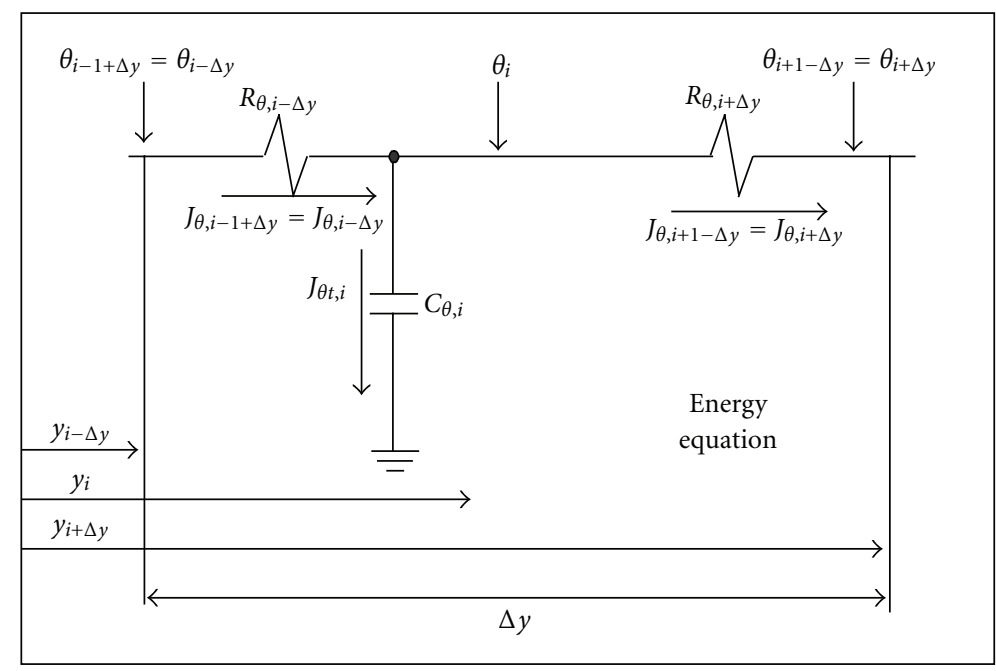

Figure 3: Electronic network model for the energy equation (2.13).

Table 1: Dimensionless spatial velocity distribution $(u)$ for $G_{r}=2.0 K_{r}=1.0, K_{p}=1.0, P_{r}=0.7$, and $t=0.2$ for the effect of Hartmann number $\left(M^{2}\right)$.

\begin{tabular}{cccc}
\hline$y$ & $M^{2} 5.0$ Laplace/NSM & $M^{2} 8.0$ Laplace/NSM & $M^{2} 10.0$ Laplace/NSM \\
\hline 0.0 & $1.0 / 1.0$ & $1.0 / 1.0$ & $1.0 / 1.0$ \\
0.5 & $0.38047 / 0.35236$ & $0.29907 / 0.28788$ & $0.26028 / 0.25409$ \\
1.0 & $0.13844 / 0.10987$ & $0.09607 / 0.08472$ & $0.07858 / 0.07234$ \\
1.5 & $0.04490 / 0.03069$ & $0.02992 / 0.02458$ & $0.02424 / 0.02158$ \\
2.0 & $0.01205 / 0.00813$ & $0.00800 / 0.00694$ & $0.00651 / 0.00627$ \\
\hline
\end{tabular}

Table 2: Dimensionless shear stress $\left(\partial u /\left.\partial y\right|_{y=0}\right)$ for $G_{r}=2.0, K_{p}=1.0, P_{r}=0.7$, and $t=0.2$ for the combined effects of radiation-conduction parameters $\left(K_{r}\right)$ and Hartmann number $\left(M^{2}\right)$.

\begin{tabular}{lccc}
\hline$M^{2}$ & $K_{r} 0.5$ Laplace/NSM & $K_{r} 1.0$ Laplace/NSM & $K_{r} 2.0$ Laplace/NSM \\
\hline 5.0 & $-1.99207 /-2.04602$ & $-1.94960 /-2.0252$ & $-1.89922 /-1.9960$ \\
8.0 & $-2.56726 /-2.58341$ & $-2.53894 /-2.56581$ & $-2.50535 /-2.54190$ \\
10.0 & $-2.90074 /-2.90404$ & $-2.87757 /-2.8840$ & $-2.85009 /-2.86732$ \\
12.0 & $-3.20605 /-3.20051$ & $-3.18645 /-3.18633$ & $-3.16320 /-3.16505$ \\
\hline
\end{tabular}

This trend is consistent with many classical studies on magneto-convection showing that the hydromagnetic body force retards the flow that is, decelerates the fluid causing a thinning in the boundary layer thickness. Very high Hartmann numbers (i.e., $M^{2} \gg 1$ ) are usually associated with the formation of a Hartmann boundary layer [39]. In Table 2, the shear stress, $\partial u /\left.\partial y\right|_{y=0}$, is found to be decreased significantly with an increase in Hartmann number $\left(M^{2}\right)$ from 5.0 through 8.0, 10.0 to 12.0, for all values of the radiation-conduction parameter, $K_{r}$. In all cases, the shear stresses are negative since the high values of Hartmann number, $M^{2}$, retard the flow in the boundary layer to such an extent that reversal of the flow is caused. This result is significant in the design of, for example, MHD generators since a critical magnetic 
flux density may be applied (i.e., Hartmann number) to reverse the flow dynamics during operation. The change in shear stresses with an increase in radiation-conduction parameter indicates that an increase in thermal radiation has a positive effect on the flow that is, reduces the degree of flow reversal. For example, for $M^{2}=5.0$, shear stress, $\partial u /\left.\partial y\right|_{y=0}$ increases (for the NSM solutions) from -2.04602 for $K_{r}=0.5$ (thermal conduction exceeds thermal radiation) to -2.0252 for $K_{r}=1.0$ (for which the thermal radiation and thermal conduction mode contributions are approximately the same), to -1.9960 for $K_{r}=2.0$ (thermal radiation exceeds thermal conduction). Therefore, for very high-strength magnetic field operating conditions, thermal radiation mitigates to some extent flow reversal effects. In Figure 4, the dimensionless shear stress profiles in time for different Hartmann numbers $\left(M^{2}\right)$ are illustrated. A strong decrease is observed in shear stress from $t=0$ to $t=0.05$, after which profiles, although they continue to decrease with increasing $M^{2}$ values, tend for $M^{2}=0,5,10$, and 15 , to the steady state. For these values of Hartmann number, profiles are always positive indicating that flow reversal does not occur. Comparing with the trends in Table 2, we can note that while the values of $K_{r}, K_{p}$, and $P_{r}$ are identical for the third column, the Grashof number $\left(G_{r}\right)$ is lower in Table 2 at 2.0, compared with the value of 10.0 in Figure 4 . As such the flow is more strongly assisted by buoyancy forces in Figure 4 which prevents the reversal of flow for all the profiles, with the exception of $M^{2}=20$ (very strong transverse magnetic field) which becomes negative for $t>0.4$, Thermal buoyancy force, $+G_{r} \theta$, is directly proportional to the Grashof free convection parameter $\left(G_{r}\right)$ and therefore would appear to assist the flow, whereas magnetic field inhibits flow acceleration in the regime. In Figure 5, temperature gradient profiles in time for the influence of radiation-conduction parameter $\left(K_{r}\right)$ are presented. In all cases, profiles are a maximum initially at the isothermal plate and decay quickly from the wall with time. An increase in $K_{r}$ from 0.1 through $0.5,1,1.5$ to 2.0 is seen to markedly reduce heat transfer gradient especially at shorter times $(0<t<0.2)$; with further elapse of time all profiles converge that is, radiation effects are negligible for large times. Increasing $K_{r}$ implies a greater augmentation of heat transfer by thermal radiation which will serve to increase fluid temperatures in the regime; the spatial heat transfer rate $\partial \theta /\left.\partial y\right|_{y=0}$ that is, temperature gradient at the wall will therefore be reduced as greater thermal energy (heat) will be imparted to the fluid-saturated regime raising temperatures within the porous regime. In Figure 6, the influence of the Darcian drag force parameter, $K_{p}$, on the time evolution of shear stress profiles, is depicted. As $K_{p}$ increases from 0.1 through $0.5,1,2$ to 5 , a very large escalation in Darcian drag force is caused, as expressed in (2.12) in the linear term, $-K_{p}^{2}$, which decelerates the flow and reduces the shear stress at the plate. Steady-state values are achieved faster with lower Darcian drag $\left(K_{p}=0.1\right)$ than with higher Darcian drag $\left(K_{p}=5\right)$. The effect of Grashof number on velocity gradient (i.e., shear stress) through time is presented in Figure 7. Increasing $G_{r}$ for the case of very low Darcian drag (i.e., highly permeable medium, $K_{p}=0.1$ ) strongly increases shear stress values at the wall that is, accelerates the flow over time. We note that values become negative for very low $G_{r}$ values since the magnetic impedance force $\left(M^{2}=5.0\right)$ will dominate and have a greater inhibiting influence with low buoyancy that is, flow reversal accompanies lower thermal buoyancy forces for higher permeability regimes. In Figure 8, the effect of the Prandtl number $\left(P_{r}\right)$ on the temporal shear stress distribution is shown again for $G_{r}=10.0, K_{r}=2.0, M^{2}=5.0$ but with $K_{p}=1.0$. Increasing $P_{r}$ strongly boosts the flow and increases shear stress profile values which remain positive for small times; however, with increasing elapse of time shear stress, values become negative indicating backflow occurs at the plate. For lower $P_{r}$ values $(0.7,0.1)$, negative values are attained more quickly that is, backflow takes place quicker. In Figure 9 , an increase in $P_{r}$ is observed to the enhance temperature gradient. Prandtl number 
controls the relative thickness of the momentum and thermal boundary layers. When $P_{r}$ is of low value, heat diffusion exceeds momentum diffusion. For $P_{r}<1$, the thickness of the thermal boundary layer therefore exceeds the thickness of the velocity boundary layer that is, temperatures will be greater. In Figure 10, temperatures are seen to decrease considerably with an increase in $P_{r}$ values (for a fixed time, $t=0.25$ ) as we progress into the boundary layer regime; profiles also decay much more sharply for higher $P_{r}$ values since momentum diffusion exceeds energy (heat) diffusion for $P_{r}>1$. For the case of $P_{r}=1$, the boundary layer thicknesses will be approximately of the same order of magnitude. For $P_{r}=0.1$, the profile is approximately linear for a substantial distance from the plate. Spatial velocity $(u)$ distributions, for two time values are illustrated in Figure 11, for the effect of Hartmann hydromagnetic parameter $\left(M^{2}\right)$. This parameter represents the ratio of the hydromagnetic retarding force to the viscous hydrodynamic force in the boundary layer. The classical velocity overshoot is identified $[1,2,4,39]$ near the moving plate surface for lower values of $M^{2}$ that is, 1.0 and 5.0; with $M^{2}=10.0$ this overshoot is clearly suppressed owing to stronger resistance to the flow. We note that for $t=1.0$, the profiles are always greater in value than for $t=0.25$ that is, the flow is accelerated considerably with time, although velocities are strongly reduced with an increase in Hartmann number. All profiles decrease towards zero in the free stream, although this state is attained much faster for higher magnetic field values $\left(M^{2}=10\right)$ and for shorter times. In Figure 12, the combined effects of time $(t)$ and radiationconduction parameter $\left(K_{r}\right)$ on spatial distribution of temperature $(\theta)$ through the boundary layer is shown. An increase in $K_{r}$ serves to supplement fluid thermal conductivity with radiation contribution and significantly heats the fluid-saturated regime that is, increases temperature values. Similar results were reported by Ali et al. [26], Hossain et al. [28], and very recently by Ghosh and Bég [41]. A large difference is observed between the profiles computed at $t=1.0$ and $t=0.25$, indicating that thermal radiation effects are amplified at greater times, compared with smaller times where the flow is still developing. After greater times a greater quantity of thermal energy will be absorbed into the fluid regime via the imposed flux causing enhanced heating of the fluid. For example, for $t=1.0$, at $y=2$, for $K_{r}=5$ (maximum thermal radiation effect), $\theta$ reaches a value of approximately 0.65 , whereas the corresponding value for $t=0.25$ is much lower at 0.35 . Finally in Figure 13 we have plotted the spatial variation of velocity for the combined effects of radiation-conduction parameter $\left(K_{r}\right)$ and time $(t)$. Again a velocity overshoot is observed in the close vicinity of the plate; however, this overshoot is distinctly greater for the highest value of $K_{r}(=2.0)$ and greater time values $(t=1.0)$; all profiles descend gradually to zero far from the wall. Thermal radiation therefore augments the flow that is, accelerates the flow in the porous regime. Velocities are minimized when thermal conduction swamps thermal radiation that is, for $K_{r}=0.1$.

\section{Conclusions}

Closed form and numerical (NSM) solutions have been presented for the transient hydromagnetic natural convection boundary layer flow past a moving vertical plane adjacent to a Darcian porous regime with thermal radiation flux present. It has been shown that thermal radiation strongly increases fluid temperatures and accelerates the flow; conversely magnetic field as simulated via the Hartmann number serves to impede the flow and reduce velocity gradient (shear stress) values. The effects of both parameters are enhanced with a greater elapse of time. Darcian drag is seen to decelerate the flow, whereas increasing free convection serves to accelerate the flow owing to the assistance of thermal buoyancy forces in the regime. 


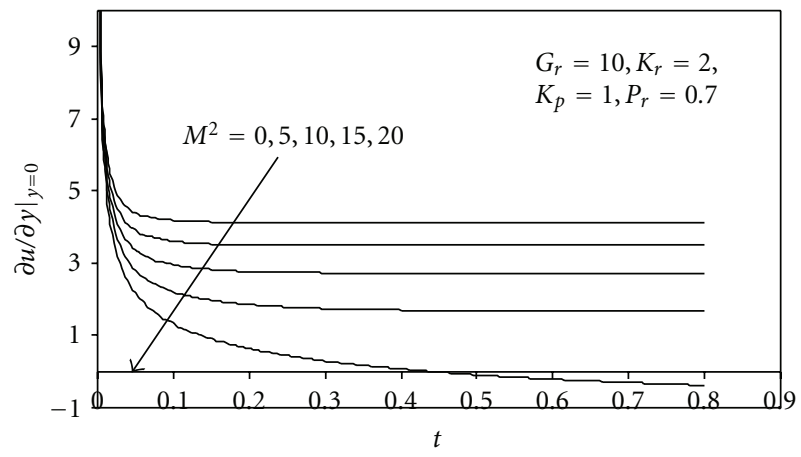

Figure 4: Temporal shear stress distribution $\left(\partial u /\left.\partial y\right|_{y=0}\right)$ with $G_{r}=10.0, K_{r}=2.0, K_{p}=1.0$, and $P_{r}=0.7$ for the effect of Hartmann hydromagnetic parameter $\left(M^{2}\right)$.

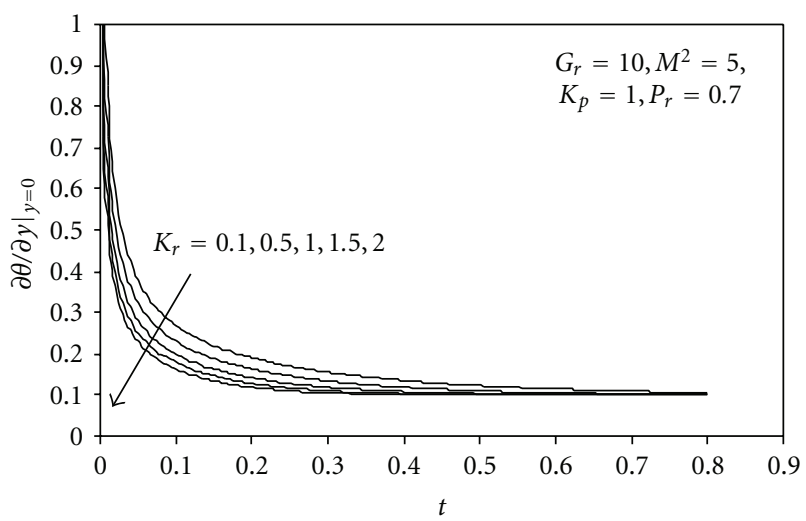

Figure 5: Temporal temperature gradient distribution $\left(\partial \theta /\left.\partial y\right|_{y=0}\right)$ with $G_{r}=10.0, M^{2}=5, K_{p}=1.0$, and $P_{r}=0.7$ for the effect of radiation-conduction number $\left(K_{r}\right)$.

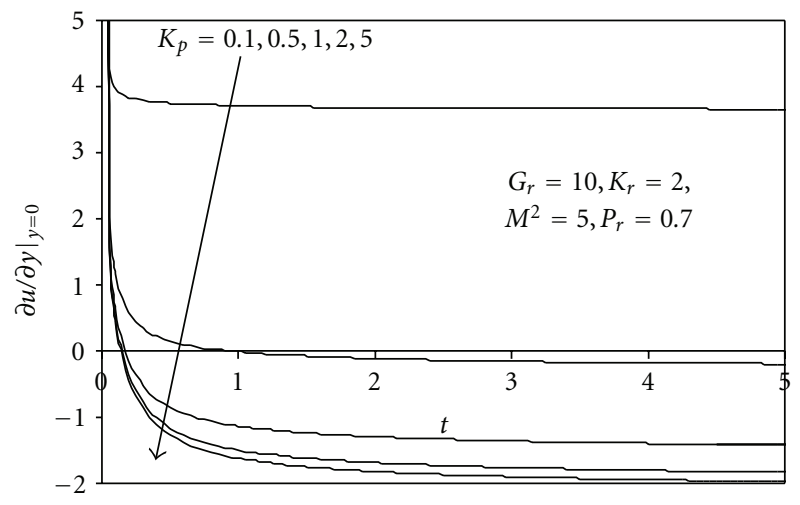

Figure 6: Temporal shear stress distribution $\left(\partial u /\left.\partial y\right|_{y=0}\right)$ with $G_{r}=10.0, M^{2}=5, K_{r}=2.0$, and $P_{r}=0.7$ for the effect of Darcian drag parameter $\left(K_{p}\right)$. 


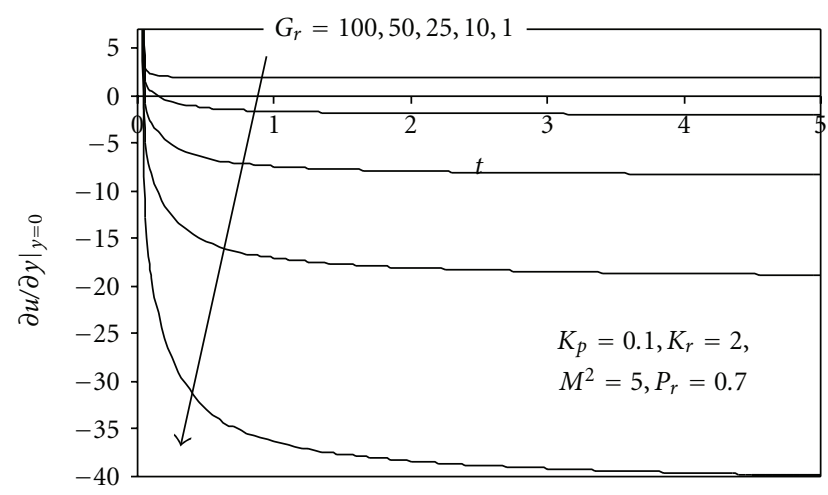

Figure 7: Temporal shear stress distribution $\left(\partial u /\left.\partial y\right|_{y=0}\right)$ with $K_{p}=0.1, M^{2}=5, K_{r}=2.0$, and $P_{r}=0.7$ for the effect of the Grashof number $\left(G_{r}\right)$.

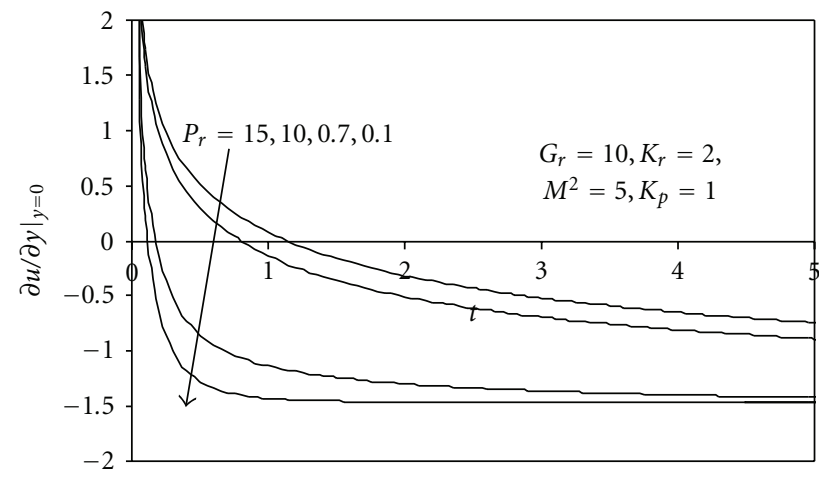

Figure 8: Temporal shear stress distribution $\left(\partial u /\left.\partial y\right|_{y=0}\right)$ with $K_{p}=1, M^{2}=5, K_{r}=2.0$, and $G_{r}=10.0$, for the effect of Prandtl number $\left(P_{r}\right)$.

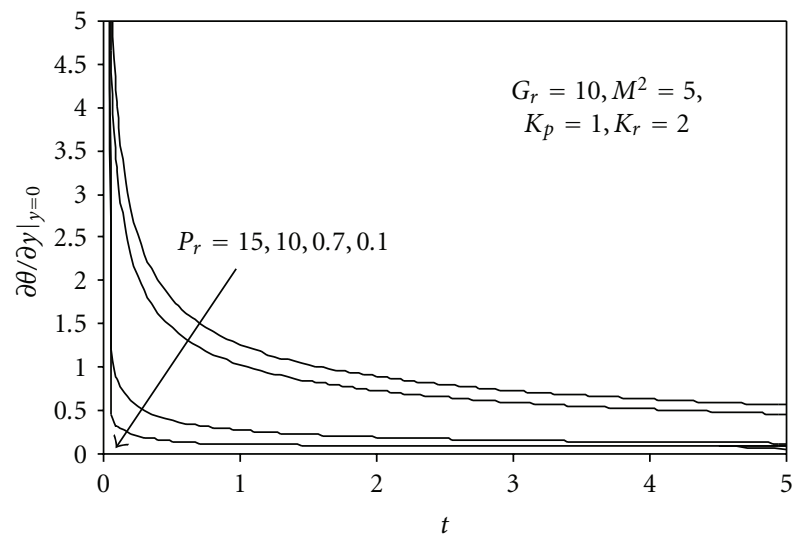

Figure 9: Temporal temperature gradient distribution $\left(\partial \theta /\left.\partial y\right|_{y=0}\right)$ with $K_{p}=1, M^{2}=5, K_{r}=2.0$, and $G_{r}=10.0$, for the effect of Prandtl number $\left(P_{r}\right)$. 


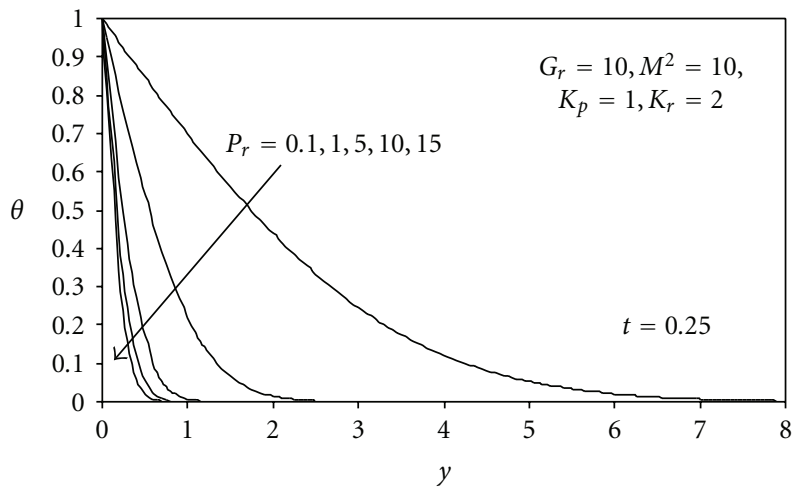

Figure 10: Spatial temperature distribution $(\theta)$ with $K_{p}=1, M^{2}=5, K_{r}=2.0$, and $G_{r}=10.0$, for the effect of Prandtl number $\left(P_{r}\right)$ at $t=0.25$.

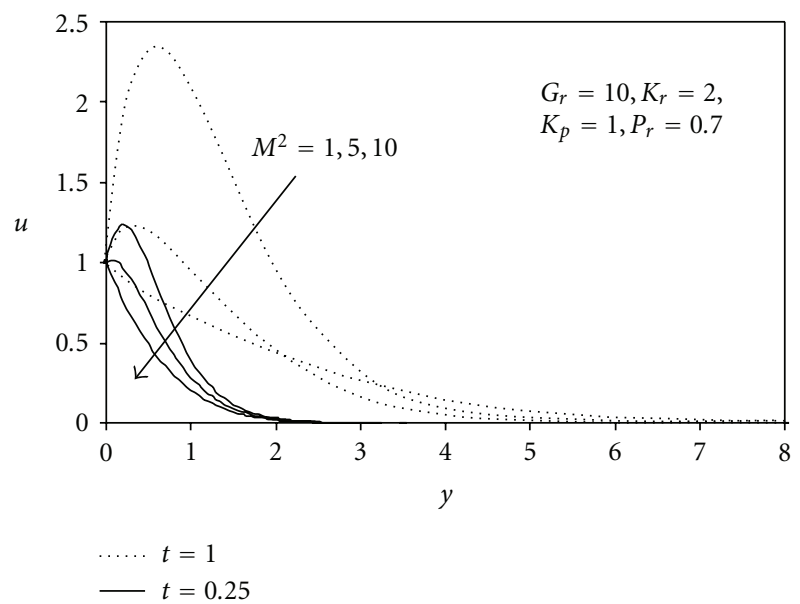

Figure 11: Spatial velocity distribution $(u)$ with $K_{p}=1, G_{r}=10, K_{r}=2.0$, and $P_{r}=0.7$ for the effect of Hartmann hydromagnetic parameter at $t=0.25$ and $t=1$.

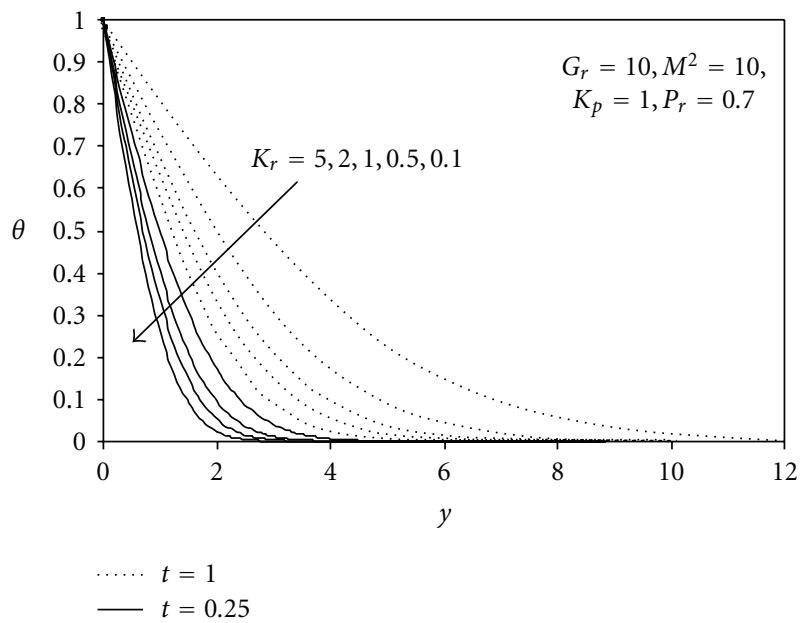

Figure 12: Spatial temperature distribution $(\theta)$ with $K_{p}=1, G_{r}=10, M^{2}=10$, and $P_{r}=0.7$ for the effect of radiation-conduction parameter $\left(K_{r}\right)$ at $t=0.25$ and $t=1$. 


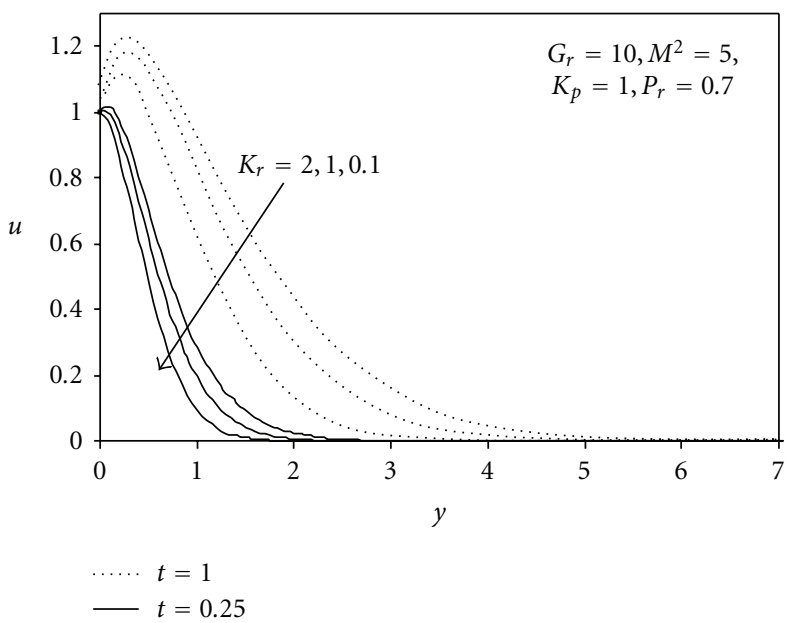

Figure 13: Spatial velocity distribution $(u)$ with $G_{r}=10.0, M^{2}=5, K_{r}=2.0$, and $P_{r}=0.7$ for the effect of radiation-conduction parameter $\left(K_{r}\right)$.

A rise in Prandtl number however decreases temperatures in the regime, but accelerates the flow that is, increases velocity gradient values. A velocity overshoot is observed with magnetic field effects but vanishes for very high values of the Hartmann number.

\section{References}

[1] G. F. Carrier and H. P. Greenspan, "The time-dependent magnetohydrodynamic flow past a flat plate," Journal of Fluid Mechanics, vol. 7, pp. 22-32, 1960.

[2] A. S. Gupta, "Steady and transient free convection of an electrically conducting fluid from a vertical plate in the presence of a magnetic field," Applied Scientific Research, vol. 9, no. 1, pp. 319-333, 1960.

[3] R. M. Singer, "Transient magnetohydrodynamic flow and heat transfer," Zeitschrift für Angewandte Mathematik und Mechanik, vol. 16, no. 4, pp. 483-494, 1965.

[4] I. Pop, "Unsteady hydromagnetic free convection flow from a vertical infinite flat plate," Zeitschrift für Angewandte Mathematik und Mechanik, vol. 49, no. 12, pp. 756-757, 1969.

[5] C. P. Yu and H. K. Yang, "Effect of wall conductances on convective magnetohydrodynamic channel flow," Applied Scientific Research, vol. 20, no. 1, pp. 16-24, 1969.

[6] V. V. R. Rao, "Transient hydromagnetic free-convection flow," Indian Journal of Physics, vol. 45, no. 5, pp. 233-235, 1971.

[7] M. Y. Antimirov and A. A. Kolyshkin, "Unsteady MHD convection in a vertical circular channel," Magnetohydrodynamics, vol. 14, no. 3, pp. 279-284, 1978.

[8] S. Rajaram and C. P. Yu, "MHD channel flow in a transient magnetic field," in ASME Symposium Forum on Unsteady Flow, 1984, Publication no. G00259.

[9] J. N. Tokis, "Unsteady magnetohydrodynamic free-convection flows in a rotating fluid," Astrophysics and Space Science, vol. 119, no. 2, pp. 305-313, 1986.

[10] S. K. Ghosh, "Unsteady hydromagnetic flow in a rotating channel with oscillating pressure gradient," Journal of the Physical Society of Japan, vol. 62, no. 11, pp. 3893-3903, 1993.

[11] N. C. Sacheti, P. Chandran, and A. K. Singh, "An exact solution for unsteady magnetohydrodynamic free convection flow with constant heat flux," International Communications in Heat and Mass Transfer, vol. 21, no. 1, pp. 131-142, 1994.

[12] H. A. Attia, "Transient MHD flow and heat transfer between two parallel plates with temperature dependent viscosity," Mechanics Research Communications, vol. 26, no. 1, pp. 115-121, 1999.

[13] M. A. Al-Nimr and M. K. Alkam, "Magnetohydrodynamics transient free convection in open-ended vertical annuli," Journal of thermophysics and heat transfer, vol. 13, no. 2, pp. 256-265, 1999. 
[14] H. S. Takhar, A. J. Chamkha, and G. Nath, "Unsteady flow and heat transfer on a semi-infinite flat plate with an aligned magnetic field," International Journal of Engineering Science, vol. 37, no. 13, pp. 1723-1736, 1999.

[15] A. T. Eswara, S. Roy, and G. Nath, "Unsteady MHD forced flow due to a point sink," Acta Mechanica, vol. 145, no. 1-4, pp. 159-172, 2000.

[16] A. J. Chamkha, "Transient hydromagnetic three-dimensional natural convection from an inclined stretching permeable surface," Chemical Engineering Journal, vol. 76, no. 2, pp. 159-168, 2000.

[17] B. K. Jha, "Natural convection in unsteady MHD couette flow," Heat and Mass Transfer, vol. 37, no. 4-5, pp. 329-331, 2001.

[18] M. A. Seddeek, "Effects of radiation and variable viscosity on a MHD free convection flow past a semiinfinite flat plate with an aligned magnetic field in the case of unsteady flow," International Journal of Heat and Mass Transfer, vol. 45, no. 4, pp. 931-935, 2001.

[19] M. Zakaria, "Magnetohydrodynamic unsteady free convection flow of a couple stress fluid with one relaxation time through a porous medium," Applied Mathematics and Computation, vol. 146, no. 2-3, pp. 469-494, 2003.

[20] S. K. Ghosh and I. Pop, "Hall effects on unsteady hydromagnetic flow in a rotating system with oscillating pressure gradient," International Journal of Applied Mechanics and Engineering, vol. 8, no. 1, pp. 43-56, 2003.

[21] J. Z. Jordán, "Numerical study of an unsteady free convective magnetohydrodynamic flow of a dissipative fluid along a vertical plate subject to a constant heat flux," International Journal of Engineering Science, vol. 44, no. 18-19, pp. 1380-1393, 2006.

[22] O. Bég, H. S. Anwar, G. N. Takhar, and A. J. Chamkha, "Mathematical Modeling of hydromagnetic convection from a rotating sphere with impulsive motion and buoyancy effects," Nonlinear Analysis: Modelling and Control, vol. 11, no. 3, pp. 227-245, 2006.

[23] H. M. Duwairi, R. A. Damseh, and B. Tashtoush, "Transient non-boussinesq magnetohydrodynamic free convection flows over a vertical surface," International Journal of Fluid Mechanics Research, vol. 33, no. 2, pp. 137-152, 2006.

[24] C. Bozkaya and M. Tezer-Sezgin, "Boundary element solution of unsteady magnetohydrodynamic duct flow with differential quadrature time integration scheme," International Journal for Numerical Methods in Fluids, vol. 51, no. 5, pp. 567-584, 2006.

[25] R. Siegel and J. R. Howell, “Thermal radiation heat transfer," Hemisphere, 1993.

[26] M. M. Ali, T. S. Chen, and B. F. Armaly, "Natural convection radiation interaction in boundary layer flow over horizontal surfaces," AIAA Journal, vol. 22, no. 12, pp. 1797-1803, 1984.

[27] A. Raptis and C. V. Massalas, "Magnetohydrodynamic flow past a plate by the presence of radiation," Heat and Mass Transfer, vol. 34, no. 2-3, pp. 107-109, 1998.

[28] M. A. Hossain, D. A. S. Rees, and I. Pop, "Free convection-radiation interaction from an isothermal plate inclined at a small angle to the horizontal," Acta Mechanica, vol. 127, no. 1-4, pp. 63-73, 1998.

[29] M. A. Abd El-Naby, E. M. E. Elbarbary, and N. Y. Abdelazem, "Finite difference solution of radiation effects on MHD unsteady free-convection flow over vertical plate with variable surface temperature," Journal of Applied Mathematics, vol. 2003, no. 2, pp. 65-86, 2003.

[30] A. Ogulu and S. Motsa, "Radiative heat transfer to magneto-hydrodynamic couette flow with variable wall temperature," Physica Scripta, vol. 71, no. 4, pp. 336-339, 2005.

[31] M. Abd-El Aziz, "Thermal radiation effects on magnetohydrodynamic mixed convection flow of a micropolar fluid past a continuously moving semi-infinite plate for high temperature differences," Acta Mechanica, vol. 187, no. 1-4, pp. 113-127, 2006.

[32] A. Ogulu and J. Prakash, "Heat transfer to unsteady magneto-hydrodynamic flow past an infinite moving vertical plate with variable suction," Physica Scripta, vol. 74, no. 2, pp. 232-239, 2006.

[33] V. R. Prasad, N. B. Reddy, and Muthucumaraswamy, "Transient radiative hydromagnetic free convection flow past an impulsively started vertical plate with uniform heat and mass flux," Theoretical Applied Mechanics, vol. 33, no. 1, pp. 31-63, 2006.

[34] J. Zueco, "Network simulation method applied to radiation and viscous dissipation effects on MHD unsteady free convection over vertical porous plate," Applied Mathematical Modelling, vol. 31, no. 9, pp. 2019-2033, 2007.

[35] A. J. Chamkha, “Unsteady hydromagnetic natural convection in a fluid-saturated porous medium channel," Advanced Filtration and Separation Technology, vol. 10, pp. 369-375, 1996.

[36] A. J. Chamkha, "Unsteady laminar hydromagnetic flow and heat transfer in porous channels with temperature-dependent properties," International Journal of Numerical Methods for Heat and Fluid Flow, vol. 11, no. 5-6, pp. 430-448, 2001. 
[37] O. A. Bég, H. S. Takhar, and A. K. Singh, "Multiparameter perturbation analysis of unsteady oscillatory magnetoconvection in porous media with heat source effects," International Journal of Fluid Mechanics Research, vol. 32, no. 6, pp. 635-661, 2005.

[38] R. C. Chaudhary and A. Jain, "Magnetohydrodynamic transient convection flow past a vertical surface embedded in a porous medium with oscillating temperature," Turkish Journal of Engineering and Environmental Sciences, vol. 32, no. 1, pp. 13-22, 2008.

[39] J. A. Shercliff, A Textbook of Magnetohydrodynamics, Pergamon, Oxford, UK, 1965.

[40] V. P. Isachenko, V. A. Osipova, and A. S. Sukomel, Heat Transfer, Mir, Moscow, Russia, 1969.

[41] S. K. Ghosh and O. A. Bég, "Theoretical analysis of radiative effects on transient free convection heat transfer past a hot vertical surface in porous media," Nonlinear Analysis: Modelling and Control, vol. 13, no. 4, pp. 419-432, 2008.

[42] J. Zueco, "Unsteady conjugate problem of a dissipative fluid in a horizontal channel with a periodic variation temperature," Meccanica, vol. 43, no. 1, pp. 37-46, 2008.

[43] O. A. Bég, H. S. Takhar, J. Zueco, A. Sajid, and R. Bhargava, "Transient Couette flow in a rotating nonDarcian porous medium parallel plate configuration: network simulation method solutions," Acta Mechanica, vol. 200, no. 3-4, pp. 129-144, 2008.

[44] O. A. Bég, J. Zueco, and H. S. Takhar, "Unsteady magnetohydrodynamic Hartmann-Couette flow and heat transfer in a Darcian channel with Hall current, ionslip, viscous and Joule heating effects: network numerical solutions," Communications in Nonlinear Science and Numerical Simulation, vol. 14, no. 4, pp. 1082-1097, 2009.

[45] O. A. Bég, J. Zueco, and H. S. Takhar, "Laminar free convection from a continuously-moving vertical surface in thermally-stratified non-Darcian high-porosity medium: network numerical study," International Communications in Heat and Mass Transfer, vol. 35, no. 7, pp. 810-816, 2008.

[46] Pspice 6.0. Irvine, California 92718. Microsim Corporation, 20 Fairbanks, 1994.

[47] L. W. Nagel, SPICE-A Computer Program to Simulate Semiconductor Circuits, chapter 4-6, UCB-ERL, M520, University of California, Berkeley, Calif, USA, 1998. 


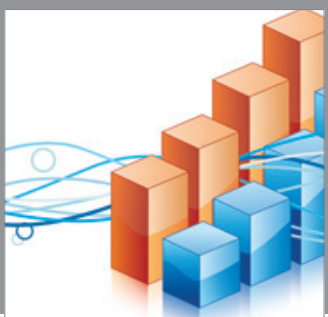

Advances in

Operations Research

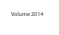

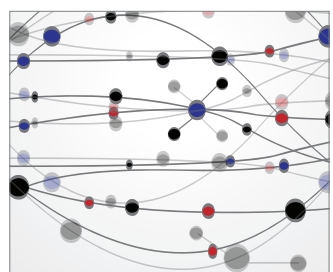

\section{The Scientific} World Journal
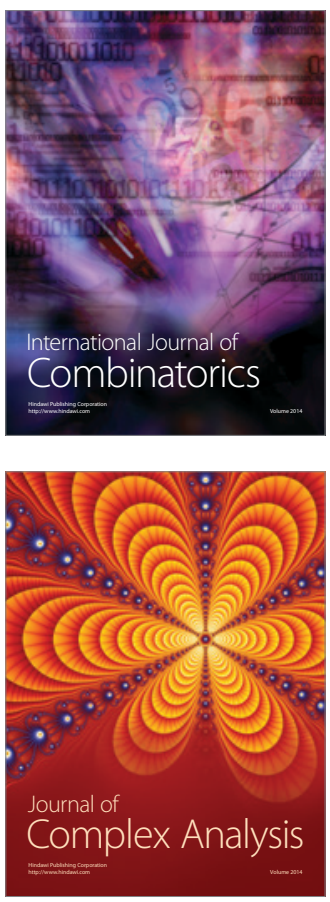

International Journal of

Mathematics and

Mathematical

Sciences
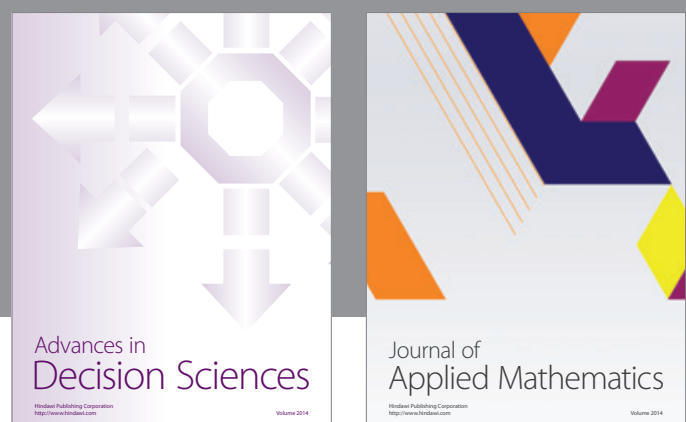

Journal of

Applied Mathematics
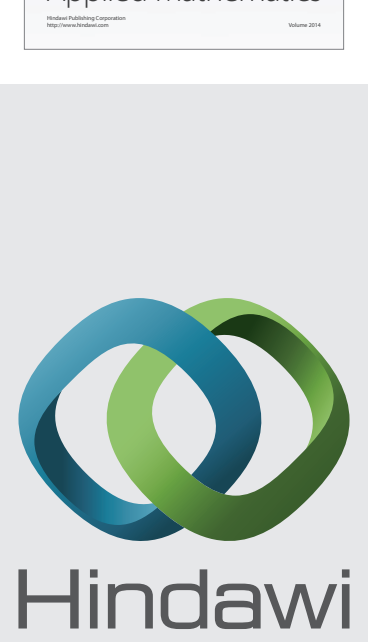

Submit your manuscripts at http://www.hindawi.com
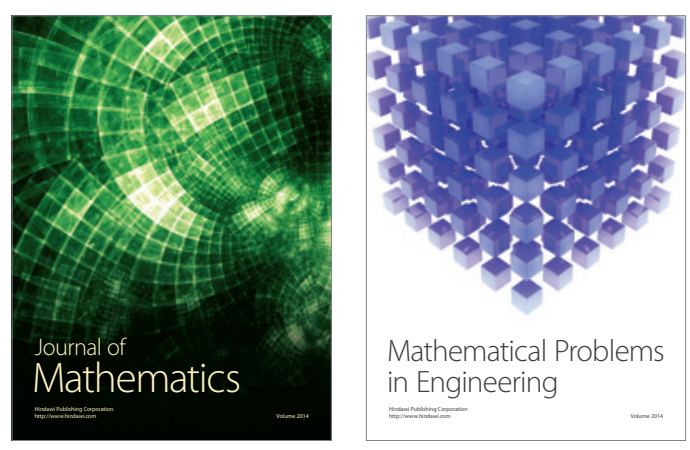

Mathematical Problems in Engineering
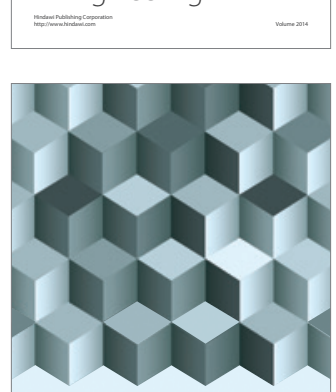

Journal of

Function Spaces
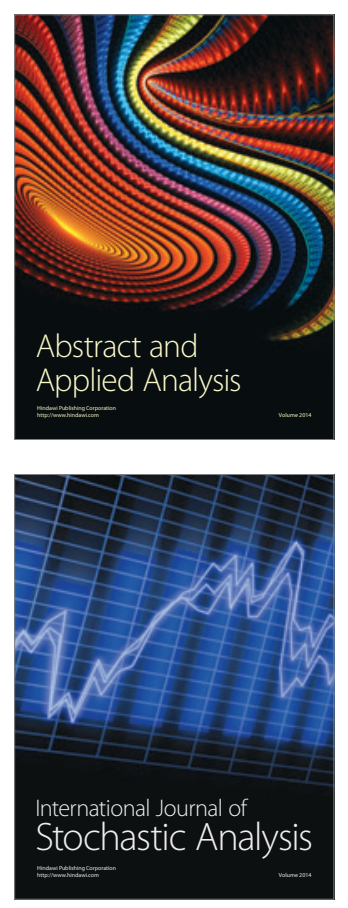

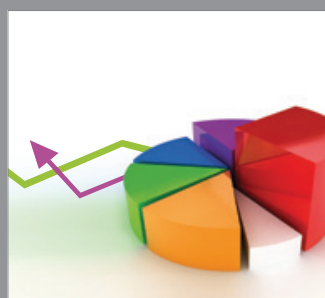

ournal of

Probability and Statistics

Promensencen
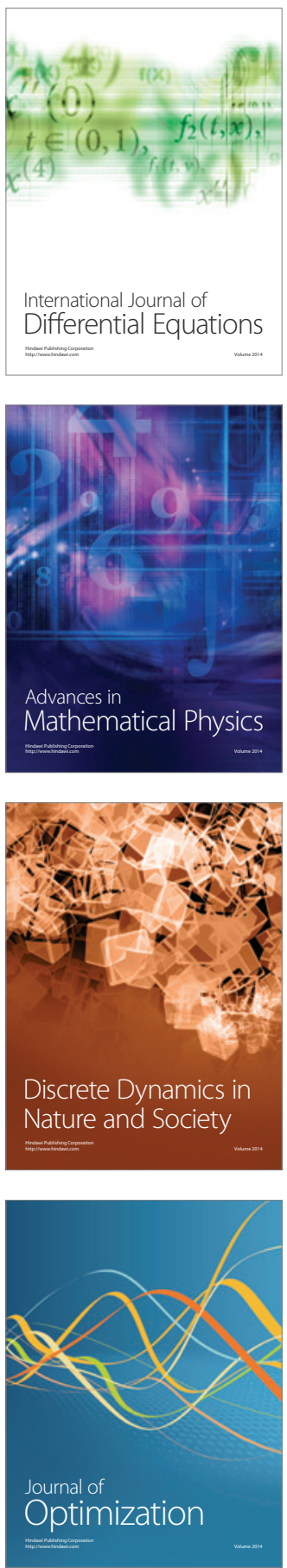